\title{
Severity of Verticillium Wilt, Plant Growth, and Spectral Reflectance Indices of Chile Pepper Under Periodic Flooding and No-flooding Conditions
}

\author{
Soumaila Sanogo ${ }^{1}$, Osama I. El-Sebai, and Robert Sanderson \\ Department of Entomology, Plant Pathology, and Weed Science, New \\ Mexico State University, N141 Skeen Hall, MSC 3BE, Las Cruces, NM \\ 88003-0003
}

\section{Additional index words. Capsicum annuum, Verticillium dahliae, soil saturation}

Abstract. Verticillium wilt, caused by Verticillium dahliae, is an economically important disease on chile pepper (Capsicum annuum $\mathrm{L}$.), which is grown primarily under furrow and drip irrigation in New Mexico. Field observations by producers indicate that Verticillium wilt is more prevalent on chile pepper grown under drip irrigation than under furrow irrigation. To test these observations, chile pepper plants at the six- to eight-leaf stage inoculated or noninoculated with $V$. dahliae were subjected to either periodic flooding or no-flooding conditions in the greenhouse. Plants under periodic flooding were subjected to three events of 7 day flooding and two events of no flooding over a period of 5 weeks. Differences among treatments were assessed using disease severity, plant dry weight, and spectral reflectance-based physiological indices such as moisture stress index (MSI), chlorophyll-based difference index, water band index, and photochemical reflectance index (PRI). There was a significant interaction between flooding and inoculation with respect to disease severity $(P<0.003)$. In plants inoculated with $V$. dahliae and subjected to periodic flooding, the relative treatment effect (RTE) based on disease severity was increased from $40 \%$ to $130 \%$ relative to noninoculated plants subjected to no flooding. However, under conditions of no flooding, RTE increased by $\approx 200 \%$ to $286 \%$ when plants were inoculated with $V$. dahliae relative to noninoculated plants. In general, periodic flooding and inoculation with $V$. dahliae were accompanied by a decrease in plant dry weight as well as in the level of spectral reflectance-based physiological indices. In noninoculated plants subjected to periodic flooding, plant dry weight was reduced by $\approx 45 \%$ to $50 \%$ relative to noninoculated plants subjected to no flooding. In plants inoculated with $V$. dahliae and subjected to periodic flooding, reduction in plant dry weight was $\approx 46 \%$ to $60 \%$ relative to noninoculated plants subjected to no flooding. Under conditions of no flooding, plant dry weight was reduced by $\approx 33 \%$ to $52 \%$ when plants were inoculated with $V$. dahliae relative to noninoculated plants. Of all the spectral reflectance-based physiological indices, PRI was the most affected by flooding and inoculation. For noninoculated plants, the mean area under the curve for PRI (AUC-PRI) was decreased by $\approx 90 \%$ under periodic flooding relative to no flooding. In plants inoculated with $V$. dahliae and subjected to periodic flooding, reduction in AUC-PRI was $\approx 93 \%$ relative to noninoculated plants subjected to no flooding. Under conditions of no flooding, AUC-PRI was reduced by $\approx 93 \%$ when plants were inoculated with $V$. dahliae relative to noninoculated plants. These results showed that chile pepper growth and physiology were affected similarly by flooding and inoculation with $\boldsymbol{V}$. dahliae. However, severity of Verticillium wilt was greater under noflooding than under periodic flooding conditions.

Chile pepper (Capsicum annuum L.) is a vegetable crop of great economic importance in the southwestern United States. Major states producing chile pepper (nonbell type) are New Mexico, California, Texas, and Arizona. In 2006, chile pepper production

Received for publication 9 Oct. 2007. Accepted for publication 14 Dec. 2007.

This research was funded by the NMSU Agricultural Experiment Station.

We thank Carmen Sanogo and Steve Thomas for reviewing the manuscript.

${ }^{1}$ To whom reprint requests should be addressed; e-mail ssanogo@nmsu.edu
3 to $7 \mathrm{~d}$ depending on soil types and environmental conditions. Furrow irrigation can be thought of as periodic flooding of fields.

Major constraints to chile pepper production in New Mexico are soilborne diseases, which are manifested by wilt symptoms after plant infection. Verticillium dahliae is a major soilborne fungal pathogen that causes wilt in several crops, including chile pepper (Pegg and Brady, 2002; Sanogo and Carpenter, 2006), in the United States and worldwide. Verticillium dahliae produces microsclerotia, which enable the pathogen to persist for at least 14 years in infested field soil (Wilhem, 1955). Additionally, this fungus survives in numerous weed species across many plant families (Sanogo and Clary, 2003; Woolliams, 1966). Verticillium dahliae affects the integrity of vascular tissues in the root and the stem. Plant infection by $V$. dahliae results in stunting, leaf chlorosis and necrosis, defoliation, vascular necrosis, wilting, and death (Pegg and Brady, 2002). After infection of pepper plants, symptoms of Verticillium wilt may appear within 3 to 6 weeks. In plants infected with $V$. dahliae, several physiological processes such as respiration, transpiration, and photosynthesis are affected, and therefore yield reduction may be incurred (Tomescu et al., 2000).

Verticillium dahliae and Phytophthora capsici, causal agent of Phytophthora blight, are the two most significant pathogens associated with chile pepper wilt in New Mexico (Sanogo and Carpenter, 2006). Verticillium dahliae and P. capsici may occur together in the same chile pepper production fields and in the same plants (Sanogo and Carpenter, 2006). The occurrence of both pathogens in the same fields represents a serious challenge to crop management. Previous studies on control of chile pepper wilt have shown that $P$. capsici could be reduced significantly by use of alternate-furrow irrigation in contrast to flood irrigation of every furrow (Biles et al., 1992) and by use of drip irrigation (Ristaino and Johnston, 1999; Xie et al., 1999). However, field observations by chile pepper producers using drip irrigation systems indicate that Verticillium wilt is increased in drip-irrigated compared with furrow-irrigated chile pepper fields. These observations were confirmed in a 3-year field survey in which Sanogo and Carpenter (2006) found that the incidence of plant infection by $V$. dahliae was $\approx 32 \%$ greater under drip irrigation than under furrow irrigation.

The cause of increased Verticillium wilt on chile pepper grown under drip irrigation relative to furrow irrigation is not known. Field notes by chile pepper producers and field surveys are observational in nature, and as such they do not provide any systematic cause-and-effect relationship to be established between irrigation and development of Verticillium wilt. There has been no systematic study to characterize chile pepper infection by $V$. dahliae and development of Verticillium wilt under drip and furrow irrigation systems. It is important to conduct a systematic evaluation of the effect of 
irrigation on Verticillium wilt on chile pepper. Such a study will be useful for crop management from at least two perspectives. First, it will provide an ecological understanding of the behavior of $V$. dahliae. Second, it will provide a knowledge base for designing strategies to minimize Verticillium wilt in chile pepper production fields.

The hypothesis of this study was that Verticillium wilt increases on chile pepper grown under drip irrigation compared with chile pepper grown under furrow irrigation. Experimentally, furrow irrigation and drip irrigation were represented by periodic flooding and no-flooding conditions, respectively. The objectives of this study were to assess the effects of periodic flooding and no-flooding conditions on severity of Verticillium wilt, plant growth, and spectral reflectance-based physiological indices of chile pepper.

\section{Materials and Methods}

Inoculum preparation and plant production. An isolate of $V$. dahliae, recovered from a field-infected chile pepper plant, was used in this study. This isolate was maintained on water agar in 9-cm diameter petri plates. Inoculum consisted of conidia, which were produced by adding a $1-\mathrm{cm}$ mycelial plug of $V$. dahliae grown on water agar to $100 \mathrm{~mL}$ of sterilized Czapek-Dox broth contained in a $250-\mathrm{mL}$ Erlenmeyer flask. All flasks were placed on a rotary shaker and incubated at room temperature ( 23 to $25^{\circ} \mathrm{C}$ ) in darkness for 7 to $10 \mathrm{~d}$, after which the contents of each flask were passed through three layers of cheesecloth to separate conidia from mycelial plugs and mats. The number of conidia in the filtered suspension was estimated with a hemacytometer and adjusted to a concentration of $4 \times 10^{6}$ conidia per milliliter.

The chile pepper cultivar 'AZ20', which is susceptible to $V$. dahliae, was used in this study. Seeds were planted in a greenhouse in six-cell plastic trays (with cell dimensions of 6,4 , and $6 \mathrm{~cm}$ in length, width, and depth, respectively) filled with sterilized Terra-Lite Metro Mix 360 (W. R. Grace \& Co., Memphis, TN). At the fourth fully expanded true leaf stage, seedlings were transplanted into plastic pots (with a diameter of $10 \mathrm{~cm}$ on top and $7.5 \mathrm{~cm}$ on bottom and a depth of $9 \mathrm{~cm}$ ) filled with sterilized TerraLite Metro Mix 360 at the rate of one seedling per pot. Seedlings were then fertilized by adding to each pot $150 \mathrm{~mL}$ solution of a $20 \% \mathrm{~N}-20 \% \mathrm{P}_{2} \mathrm{O}_{5}-20 \% \mathrm{~K}_{2} \mathrm{O}$ fertilizer (Scotts Co., Marysville, OH) containing $3.94 \%$ ammoniacal nitrogen, $6.05 \%$ nitrate, $10.01 \%$ urea, $0.05 \%$ magnesium, $0.0068 \%$ boron, $0.0036 \%$ copper (chelated $\mathrm{Cu}$ ), $0.05 \%$ iron (chelated Fe), $0.025 \%$ manganese (chelated $\mathrm{Mn}), 0.0009 \%$ molybdenum, and $0.0025 \%$ zinc (chelated $\mathrm{Zn}$ ).

Effects of periodic flooding and no flooding on infection of chile pepper by Verticillium dahliae. At the six- to eight-leaf stage, potted plants were inoculated with 0 , 5 , and $10 \mathrm{~mL}$ of conidia suspension $\left(4 \times 10^{6}\right.$ conidia $/ \mathrm{mL}$ ) to generate three inoculum levels: $0,2 \times 10^{7}$, and $4 \times 10^{7}$ conidia per pot. Conidia suspension was dispensed onto the surface of the soil in each pot $\approx 2 \mathrm{~cm}$ away from the lower stem of each plant. For seedlings serving as controls (inoculum level $=0$ ), no inoculum was dispensed on top of soil in each pot.

After inoculation, one set of plants were subjected to periodic flooding conditions in the greenhouse. Flooding conditions were established using methods outlined by Kuan and Erwin (1980) by placing pots containing plants on plant saucers $(12.5 \mathrm{~cm}$ diameter and $3.5 \mathrm{~cm}$ depth), which were kept full of tap water (electrical conductivity $=0.5 \mathrm{dS} / \mathrm{m}$ ). Periodic flooding consisted of $7 \mathrm{~d}$ of flooding followed by $7 \mathrm{~d}$ of no flooding. During the $7 \mathrm{~d}$ of no-flooding conditions, pots containing plants were removed from plant saucers, placed on the greenhouse bench, and watered as needed. Each potted plant was subjected to three events of flooding and two events of no flooding over a 5-week period. A second set of plants was maintained on the greenhouse bench, monitored daily, and irrigated as needed over the same 5-week period. No saucers were placed under potted plants in this second set and plants were irrigated by gently adding water on top of soil with a plastic beaker to avoid splash onto aboveground plant parts. Soil water potential was monitored in potted plants placed under both periodic flooding and no-flooding conditions using Watermark soil moisture sensors (Spectrum Technologies, Plainfield, IL).

The study was conducted as a $2 \times 3$ factorial experiment with six treatments generated from the combination of two flooding conditions (periodic flooding and no flooding) and three inoculum levels $\left(0,2 \times 10^{7}\right.$, and $4 \times 10^{7}$ conidia per pot). The six treatment combinations were evaluated in a split plot with flooding as whole plot factor and inoculation as subplot factor. Each treatment was replicated five times with a pot being a replicate. The entire experiment was completed twice. Expt. 1 was conducted from 18 Apr. to 23 May 2006, and Expt. 2 was carried out from 1 June to 6 July 2006.

Measurement of spectral reflectancebased physiological indices. The spectral reflectance in the $350 \mathrm{~nm}$ to $2500 \mathrm{~nm}$ range was measured on the two most fully expanded chile pepper leaves below the shoot apex using a portable field spectroradiometer, FieldSpec Pro FR (Analytical Spectral Devices, Boulder, CO), attached through a fiberoptic cable to the LI-COR 1800 integrating sphere (LI-COR 1800-12; Li-COR, Lincoln, $\mathrm{NE}$ ). An integrating sphere is designed to collect all of the radiation reflected from or transmitted through a surface. The 1800-12 is an external integrating sphere, which means that the sample is held to the outside of the sphere with a small section of the sample acting as part of the sphere wall. The interior of the sphere is coated with barium sulfate to make a uniform diffuse reflector. An internal integrating sphere would have the sample entirely inside the sphere, generally near the center. A measurement of reflectance involves comparing the wall illumination caused by a focused beam of radiation reflected from the sample material with that reflected from the white reference material. The two measurements were taken sequentially. The 1800-12 uses the same illumination for both sample and reference measurement by moving it from one port to the other between measurements. The integrating sphere was initialized following procedures given in the "RS3 Quick Reference for FieldSpec Pro." Each single leaf sample was scanned individually on the upper (adaxial) surface of the leaf, taking 50 spectra for each single leaf to reduce spectral and detector noise. The collected hyperspectral data were stored on a hard disk for subsequent viewing and analysis with software provided with the spectroradiometer (Analytical Spectral Devices, ViewSpecPro v.4.2). The measured reflectance spectra were used to calculate four physiological indices: moisture stress index (MSI), water band index (WBI), chlorophyll-based difference index (CBDI), and photochemical reflectance index (PRI). MSI is determined by the ratio of the reflectance at $1600 \mathrm{~nm}$ and reflectance at $820 \mathrm{~nm}$ (Hunt and Rock, 1989) and measures the plant water stress; the lower the value of MSI, the less stressed is a plant. WBI is the ratio between reflectance at $900 \mathrm{~nm}$ and reflectance at $970 \mathrm{~nm}$ (Gamon and Qiu, 1999; Penuelas et al., 1997) and measures the water content in the leaf; the higher the value of WBI, the higher the water content in the leaf. CBDI, which is the ratio of $(705-750) /(705$ +750 ) measures the total chlorophyll concentration; the higher the value of CBDI, the higher chlorophyll concentration in the leaf. PRI is the ratio of $(531-570) /(531+570)$ and measures the photosynthetic activity of the plant; the higher value of the PRI, the higher photosynthetic activity of the plant. Measurements of spectral reflectance were done at the end of each periodic flooding across all treatments. The area under the spectral reflectance index curve (AUC) was computed using the trapezoid method as follows:

$$
\mathrm{AUC}=\sum_{i=1}^{n-1}\left[\left(Y_{i+1}+Y_{i}\right) / 2\right] \times\left[T_{i+1}-T_{i}\right],
$$

where $Y_{i}=$ spectral reflectance index value at the $i$ th observation, $T_{i}=$ time (in days) at the $i$ th observation, and $n=$ total number of observations. AUC was used in assessing differences among treatments.

Assessment of disease severity and plant biomass. Plants were monitored for disease symptoms. At the end of the third periodic flooding ( 5 weeks after inoculation), disease severity on aboveground plant parts was evaluated using a scale modified from BejaranoAlcázar et al. (1996) and based on the percentage of foliage affected with chlorosis, necrosis, wilting, defoliation, and the presence of discoloration in vascular tissue in the stem: $0=$ no symptoms; $1=1 \%$ to $33 \%$ foliage affected; $2=1 \%$ to $33 \%$ foliage 
affected with vascular discoloration; $3=34 \%$ to $66 \%$ foliage affected; $4=34 \%$ to $66 \%$ foliage affected with vascular discoloration; $5=67 \%$ to $100 \%$ foliage affected; $6=67 \%$ to $100 \%$ foliage affected with vascular discoloration; and 7 = dead plant.

Each plant was severed at the crown and cut longitudinally to assess the presence of vascular discoloration. Roots of each plant were washed free of soil. Aboveground plant parts and roots of each plant were combined in a brown paper bag and placed in an oven at $65^{\circ} \mathrm{C}$. Plant dry weight was recorded after $48 \mathrm{~h}$.

Statistical analysis. Data on the following variables were used: 1) disease severity ratings, 2) plant dry weight, and 3) the area under the spectral reflectance index curve (AUC). Data on plant dry weight and AUC were subjected to analysis of variance using Proc Mixed procedure in SAS (SAS Institute, Cary, NC). The natural logarithm transformation was applied to data on plant dry weight before analysis of variance. For each variable, data from individual trials were analyzed separately to assess consistency of results and homogeneity of error variances among experiments. Homogeneity of error variances was evaluated following procedures outlined by Gomez and Gomez (1984). When results were consistent among experiments and error variances were homogeneous $(P>0.05)$, data from all experiments were combined. Because the data consisting of disease severity ratings were ordinal, they were analyzed by nonparametric approaches using SAS macros F1_LD_F1 (experimental design with one whole plot factor and one subplot factor) and LD_CI (limits of confidence interval) as outlined by Brunner et al. (2002). These macros allow computation of relative treatment effects (RTE), 95\% confidence intervals for RTE, and analysis of variance-type statistics for gauging the significance of main factors and the interactions among these factors. Means separation for plant dry weight and area under the reflectance index curve was based on the probability value of least squares means difference among experimental factors, which was obtained using the LSMEANS statement in SAS with the PDIFF option. Presentation of data was completed following methods outlined by Gomez and Gomez (1984).

\section{Results}

Homogeneity of error variances was not indicated for disease severity $\left(P<10^{-3}\right)$ and plant dry weight $(P=0.01)$. Therefore, data were analyzed for each experiment separately for these variables. However, homogeneity of error variances was indicated for reflectance-based physiological indices, so data on these indices were pooled across both experiments.

\section{Disease severity and plant biomass}

Expt. 1. The first symptoms of infection were observed as leaf drop on chile pepper 'AZ20' in the no-flooding treatment at the highest inoculum level by $18 \mathrm{~d}$ after inoculation. At 5 weeks after inoculation, severe wilting was observed in no-flooding treatment under both low and high inoculum levels. Conversely, plants subjected to periodic flooding displayed symptoms of slight leaf chlorosis and leaf scorching under both low and high inoculum levels. Noninoculated plants subjected to the no-flooding treatment displayed no perceptible symptoms. Conversely, slight chlorosis was visible on foliage of noninoculated plants subjected to periodic flooding.

As revealed by analysis of variance (Table 1), there was a significant interactive effect of flooding and inoculation $\left(P=10^{-5}\right)$ on disease severity. The RTEs computed for the various levels of flooding-inoculation treatments are shown in Table 2. RTE was 0.28 for noninoculated plants subjected to periodic flooding and for noninoculated plants subjected to no flooding. In plants inoculated with $V$. dahliae and subjected to periodic flooding, RTE increased by $\approx 40 \%$ relative to noninoculated plants subjected to no flooding. However, under conditions of no flooding, RTE increased by $\approx 200 \%$ when plants were inoculated with $V$. dahliae relative to noninoculated plants.

Means and SE of the means for plant dry weight are shown in Figure 1A. There was no significant interactive effect of flooding and inoculation (Table 5) on plant dry weight $(P=0.3066)$. However, there were significant main effects of flooding $(P=0.0115)$ and inoculation $(P=0.0002)$ on plant dry weight. When noninoculated plants were subjected to periodic flooding, plant dry weight was reduced by $\approx 45 \%$ relative to noninoculated plants subjected to no flooding. In plants inoculated with $V$. dahliae and subjected to periodic flooding, reduction in plant dry weight was $\approx 56 \%$ to $60 \%$ relative to noninoculated plants subjected to no flooding. Under conditions of no flooding, plant dry weight was reduced by $\approx 40 \%$ to $52 \%$ when plants were inoculated with $V$. dahliae relative to noninoculated plants.

Expt. 2. Leaf drop was the first symptom observed on chile pepper 'AZ20' at low and high inoculum level at $21 \mathrm{~d}$ after inoculation.

Table 1. Analysis of disease severity ratings on chile pepper 'AZ20' in relation to inoculation with Verticillium dahliae and flooding treatments (Expt. 1). ${ }^{\mathrm{z}}$

\begin{tabular}{lcccc}
\hline & \multicolumn{4}{c}{ ANOVA-type statistics $^{\mathrm{y}}$} \\
\cline { 2 - 5 } Factor & Fn & df1 & df2 & $P$ value \\
\hline Flooding (F) & 72.54 & 1 & 7.71 & 0.00003 \\
Inoculation (I) & 28.03 & 1.41 & $\infty$ & $<0.00001$ \\
F $\times$ I & 14.99 & 1.41 & $\infty$ & 0.00001 \\
\hline
\end{tabular}

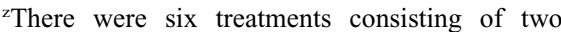
flooding conditions (periodic flooding and no flooding) and three inoculum levels $\left(0,2 \times 10^{7}\right.$, and $4 \times 10^{7}$ conidia per pot).

${ }^{\mathrm{y}} \mathrm{Fn}$ is an ANOVA-type statistic equivalent to an $F$ value with estimated degree of freedom df1 (numerator) and df2 (denominator). The significance of main factors and interactions among these factors is gauged by the $P$ values.

ANOVA $=$ analysis of variance.
At 5 weeks after inoculation, severe defoliation, wilting, and death were observed in the no-flooding treatment under both low and high inoculum levels. Noninoculated plants subjected to no-flooding treatment displayed no perceptible symptoms. Defoliation was observed on plants subjected to periodic flooding treatment under both low and high inoculum levels, albeit at a limited extent. Slight defoliation was also visible on noninoculated plants subjected to periodic flooding.

There was a significant interaction of flooding and inoculation $(P=0.0027)$ on disease severity (Table 3 ). The RTEs computed for the various flooding-inoculation levels are shown in Table 4. RTE was 0.21 for noninoculated plants subjected to the periodic flooding treatment and for noninoculated plants subjected to no flooding. For plants inoculated with $V$. dahliae and subjected to periodic flooding, RTE increased by $\approx 130 \%$ relative to noninoculated plants subjected to no flooding. Under conditions of no flooding, RTE increased by $\approx 286 \%$ when plants were inoculated with $V$. dahliae relative to noninoculated plants.

Means and SE of the means for plant dry weight are shown in Figure 1B. There was no significant interactive effect of flooding and inoculation (Table 5) on plant dry weight $(P=0.1767)$. However, there were significant main effects of flooding $(P=0.045)$ and inoculation $(P=0.0004)$ on plant dry weight. In noninoculated plants subjected to periodic flooding, plant dry weight was reduced by $\approx 50 \%$ relative to noninoculated plants subjected to no flooding. In plants inoculated with $V$. dahliae and subjected to periodic flooding, reduction in plant dry weight was $\approx 46 \%$ to $50 \%$ relative to noninoculated plants subjected to no flooding. Thus, flooding effect was not significantly exacerbated by inoculation with $V$. dahliae. Under conditions of no flooding, plant dry weight was reduced by $\approx 33 \%$ to $47 \%$ when plants were inoculated with $V$. dahliae relative to noninoculated plants. Thus, the effect of no flooding on plant dry weight in the presence of $V$. dahliae was comparable to the effect of periodic flooding on plant dry weight regardless of the absence or presence of $V$. dahliae. In essence, these results showed that flooding stress was equivalent to inoculation stress on plant growth.

\section{Spectral reflectance-based \\ physiological indices}

As shown in Table 6, analysis of variance revealed significant main and interactive effects $(0.01<P<0.0010)$ of flooding and inoculation treatments on AUC for PRI. Significant main effects were detected for flooding $(P=0.0004)$ and for inoculation $(P=0.0038)$ with respect to CBDI. However, there were neither significant main effects nor interactive effects $(P>0.6)$ of flooding and inoculation treatments on the AUC for MSI and WBI.

The mean area AUC for PRI in plants noninoculated was decreased by $\approx 90 \%$ under 
Table 2. Median, relative treatment effects (RTE), and 95\% confidence intervals (CIs) for RTE of severity ratings on chile pepper 'AZ20' in relation to inoculation with Verticillium dahliae and flooding treatments (Expt. 1).

\begin{tabular}{lcccc}
\hline Flooding $^{\mathrm{z}}$ & Inoculation $^{\mathrm{y}}$ & Median $^{\mathrm{x}}$ & RTE & 95\% CI for RTE \\
\hline Periodic flooding & 0 & 0 & 0.28 & $(0.246-0.326)$ \\
& 20 & 1 & 0.42 & $(0.293-0.557)$ \\
No flooding & 40 & 1 & 0.35 & $(0.246-0.481)$ \\
& 0 & 0 & 0.28 & $(0.246-0.326)$ \\
& 20 & 2 & 0.78 & $(0.748-0.801)$ \\
& 40 & 7 & 0.89 & $(0.847-0.907)$ \\
\hline
\end{tabular}

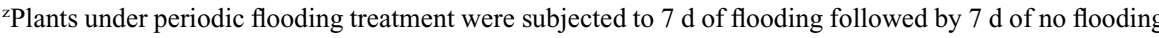
over a 5-week period. Plants subjected to no-flooding treatment were monitored daily and irrigated as needed over the same 5-week period.

${ }^{\mathrm{y}}$ There were three inoculum levels of $0,2 \times 10^{7}\left(20\right.$ million), and $4 \times 10^{7}$ (40 million) conidia per pot, which were designated 0,20 , and 40 , respectively.

${ }^{x}$ Disease severity on aboveground plant parts was evaluated using a scale based on the percentage of foliage affected with chlorosis, necrosis, wilting, defoliation, and the presence of discoloration in vascular tissue in the stem: $0=$ no symptoms; $1=1 \%$ to $33 \%$ foliage affected; $2=1 \%$ to $33 \%$ foliage affected with vascular discoloration; $3=34 \%$ to $66 \%$ foliage affected; $4=34 \%$ to $66 \%$ foliage affected with vascular discoloration; $5=67 \%$ to $100 \%$ foliage affected; $6=67 \%$ to $100 \%$ foliage affected with vascular discoloration; and $7=$ dead plant
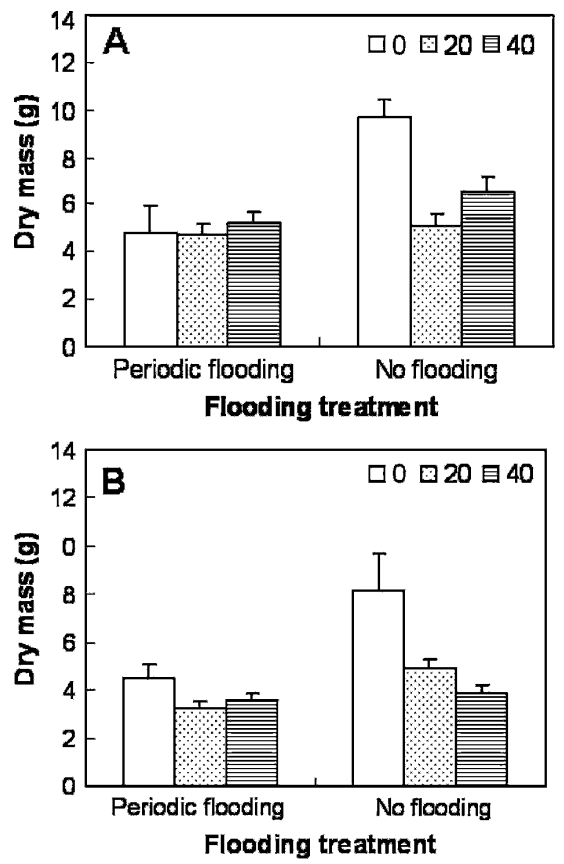

Fig. 1. Effect of periodic flooding and no flooding and inoculation of chile pepper 'AZ20' with Verticillium dahliae at three inoculum levels on plant dry weight in (A) Expt. 1 and (B) Expt. 2. Columns are the means for each treatment and bars on the columns are the SES of the means. There were three inoculum levels of $0,2 \times 10^{7}(20$ million $)$, and $4 \times 10^{7}$ (40 million) conidia per pot, which were designated 0,20 , and 40, respectively. For each experiment, there was no significant interaction between flooding treatment and inoculation $(P>0.150)$. Probability of least squares means difference $(P$-diff) between periodic flooding and no flooding was 0.0115 in Expt. 1 and 0.045 in Expt. 2. The $P$-diff values among inoculum levels $(0,20$, and 40$)$ were as follows in experiment $1: P$-diff $(0$ and 20$)$ $=0.0023 ; P$-diff $(0$ and 40$)<0.0001 ;$ and $P$-diff $(20$ and 40$)=0.1775$. The $P$-diff values among inoculum levels $(0,20$, and 40$)$ were as follows in Expt. 2: $P$-diff $(0$ and 20$)<0.0001$; $P$-diff $(0$ and 40$)=0.0073$; and $P$-diff $(20$ and 40) $=0.0476$.
Table 3. Analysis of disease severity ratings on chile pepper 'AZ20' in relation to inoculation with Verticillium dahliae and flooding treatments (Expt. 2). ${ }^{\mathrm{C}}$

\begin{tabular}{lrccc}
\hline & \multicolumn{4}{c}{ ANOVA-type statistics ${ }^{\mathrm{y}}$} \\
\cline { 2 - 5 } Factor & \multicolumn{1}{c}{ Fn } & df1 & df2 & $P$ value \\
\hline Flooding (F) & 39.29 & 1 & 7.71 & 0.00024 \\
Inoculation (I) & 26.09 & 1.66 & $\infty$ & $<0.00001$ \\
F $\times$ I & 6.58 & 1.66 & $\infty$ & 0.0027
\end{tabular}

${ }^{z}$ There were six treatments generated from the combination of two flooding conditions (periodic flooding and no flooding) and three inoculum levels $\left(0,2 \times 10^{7}\right.$, and $4 \times 10^{7}$ conidia per pot $)$.

${ }^{\mathrm{y}} \mathrm{Fn}$ is an ANOVA-type statistic equivalent to an $F$ value with estimated degree of freedom df1 (numerator) and df2 (denominator). The significance of main factors and interactions among these factors is gauged by the $P$ values.

ANOVA $=$ analysis of variance.

periodic flooding relative to no flooding (Fig. 2). In plants inoculated with $V$. dahliae and subjected to periodic flooding, reduction in AUC-PRI was $\approx 93 \%$ relative to noninoculated plants subjected to no flooding. Under conditions of no flooding, AUC-PRI was reduced by $\approx 93 \%$ when plants were inoculated with $V$. dahliae relative to noninoculated plants. Thus, the effect of no flooding on PRI in the presence of $V$. dahliae was comparable to the effect of periodic flooding on PRI regardless of the absence or presence of $V$. dahliae.

The mean AUC for CBDI was reduced by $\approx 10 \%$ in plants under periodic flooding relative to those under no-flooding conditions (Fig. 3). In plants inoculated with $V$. dahliae, the mean value of AUC-CBDI was reduced by $\approx 7 \%$ to $10 \%$ relative to noninoculated plants. Thus, the effect of periodic flooding on AUC-CBDI was comparable to the effect of inoculation on AUC-CBDI.

In both Expts. 1 and 2, soil water potential varied from -0.003 to $-0.005 \mathrm{MPa}$ in pots under periodic flooding conditions and from -0.005 to $-0.025 \mathrm{MPa}$ in pots under noflooding conditions. These values of water potential are well within those recorded with the same type of sensors in chile pepper fields fluctuating between -0.006 and $-0.017 \mathrm{MPa}$ during furrow irrigation events. Average daily air temperatures in the greenhouse ranged from 24.4 to $28.2{ }^{\circ} \mathrm{C}$ in Expt. 1, and from 23.3 to $28.3{ }^{\circ} \mathrm{C}$ in Expt. 2 over the duration of each experiment.

\section{Discussion}

This study is the first known report that systematically examined the relationship of periodic flooding and no-flooding conditions to the development of Verticillium wilt on chile pepper. Periodic flooding was shown to be less conducive to Verticillium wilt compared with no-flooding conditions where soil moisture is below the saturation level. Results from this study support the hypothesis that Verticillium wilt increases under drip irrigation compared with furrow irrigation, which were experimentally represented by no-flooding and periodic flooding conditions, respectively. These results may help to explain field observations by chile pepper producers indicating an increased level of Verticillium wilt under drip irrigation compared with furrow irrigation.

The conditions imposed in this study were assumed to be representative of those encountered under a field environment in drip-irrigated and furrow-irrigated fields. The method of flooding was comparable to that used in other studies (Kuan and Erwin, 1980) and was assumed to mimic moisture level that would be found under field conditions. The scope of this study was limited to three periodic events during a 5-week period of active vegetative growth of chile pepper. However, it is recognized that more than three periodic flooding events may take place in production fields. Additionally, it must be realized that flooding duration may be less than the level selected in this work. Within the scope of this study, it was established that Verticillium wilt was enhanced under noflooding conditions in contrast to periodic flooding.

Several methods of plant inoculation and types of inoculum have been used in plant infection studies with Verticillium dahliae across many crops. Plant inoculation methods included root-dip with or without root trimming (Bhat and Subbarao, 1999; Tsor et al., 1998), soil infestation (Riggs and Graham, 1995; Riley and Bosland, 1998), and stem injection (DeVay et al., 1997). In the root-dip method, plants may need to be uprooted, dipped in inoculum suspension, and transplanted. Plant response to infection may be affected as a result of wounding generated during uprooting and transplant shock. Stem injection may modify plant response by generating wounds and impede assessment of the interaction between host plant and pathogen at the host interface. Soil infestation was used in this study because this method does not affect the physiological integrity of plants.

Inoculum types used in several studies consisted of conidia (Bhat and Subbarao, 1999; Riggs and Graham, 1995; Tsor et al., 1998) and microsclerotia (Farley et al., 1970; 
Table 4. Median, relative treatment effects (RTE), and 95\% confidence intervals (CIs) for RTE of severity ratings on chile pepper 'AZ20' in relation to inoculation with Verticillium dahliae and flooding treatments (Expt. 2).

\begin{tabular}{lcccc}
\hline Flooding $^{z}$ & Inoculation $^{y}$ & Median $^{\mathrm{x}}$ & RTE & 95\% CI for RTE \\
\hline Periodic flooding & 0 & 0 & 0.21 & $(0.141-0.344)$ \\
& 20 & 3 & 0.55 & $(0.376-0.713)$ \\
No flooding & 40 & 3 & 0.41 & $(0.301-0.533)$ \\
& 0 & 0 & 0.21 & $(0.129-0.368)$ \\
& 20 & 6 & 0.72 & $(0.644-0.774)$ \\
& 40 & 7 & 0.90 & $(0.807-0.914)$ \\
\hline
\end{tabular}

zPlants under periodic flooding treatment were subjected to $7 \mathrm{~d}$ of flooding followed by $7 \mathrm{~d}$ of no flooding over a 5-week period. Plants subjected to no-flooding treatment were monitored daily and irrigated as needed over the same 5 -week period.

y There were three inoculum levels of $0,2 \times 10^{7}(20$ million $)$, and $4 \times 10^{7}$ (40 million) conidia per pot, which were designated 0,20 , and 40 , respectively.

${ }^{x}$ Disease severity on aboveground plant parts was evaluated using a scale based on the percentage of foliage affected with chlorosis, necrosis, wilting, defoliation, and the presence of discoloration in vascular tissue in the stem: $0=$ no symptoms; $1=1 \%$ to $33 \%$ foliage affected; $2=1 \%$ to $33 \%$ foliage affected with vascular discoloration; $3=34 \%$ to $66 \%$ foliage affected; $4=34 \%$ to $66 \%$ foliage affected with vascular discoloration; $5=67 \%$ to $100 \%$ foliage affected; $6=67 \%$ to $100 \%$ foliage affected with vascular discoloration; and $7=$ dead plant.

Table 5. Analysis of plant dry weight measured on chile pepper 'AZ20' in relation to inoculation with Verticillium dahliae and flooding treatments. ${ }^{\mathrm{z}}$

\begin{tabular}{lll}
\hline & \multicolumn{2}{c}{$P$ value $^{\mathrm{y}}$} \\
\cline { 2 - 3 } Factor & Expt. 1 & Expt. 2 \\
\hline Flooding (F) & 0.0115 & 0.045 \\
Inoculation (I) & 0.0002 & 0.0004 \\
$\mathrm{~F} \times \mathrm{I}$ & 0.3066 & 0.1767 \\
\hline
\end{tabular}

zThere were six treatments generated from the combination of two flooding conditions (periodic flooding and no flooding) and three inoculum levels $\left(0,2 \times 10^{7}\right.$, and $4 \times 10^{7}$ conidia per pot).

${ }^{\mathrm{y}}$ The significance of main factors and interactions among these factors is gauged by the $P$ values.

Table 6. Analysis of the area under the index curve for moisture stress index (MSI), water band index (WBI), chlorophyll-based difference index (CBDI), and photochemical reflectance index (PRI) measured on chile pepper 'AZ20' in relation to inoculation with Verticillium dahliae and flooding treatments. ${ }^{\mathrm{z}}$

\begin{tabular}{lcccc}
\hline & \multicolumn{4}{c}{$P$ value $^{\mathrm{y}}$} \\
\cline { 2 - 5 } Factor & MSI & WBI & CBDI & PRI \\
\hline Flooding (F) & 0.6336 & 0.9107 & 0.0004 & 0.0117 \\
Inoculation (I) & 0.8304 & 0.9895 & 0.0038 & 0.0008 \\
F $\times$ I & 0.9567 & 0.9883 & 0.4075 & 0.0160 \\
\hline
\end{tabular}

zThere were six treatments generated from the combination of two flooding conditions (periodic flooding and no flooding) and three inoculum levels $\left(0,2 \times 10^{7}\right.$, and $4 \times 10^{7}$ conidia per pot $)$. Data from Expts. 1 and 2 were combined.

${ }^{\mathrm{y}}$ The significance of main factors and interactions among these factors is gauged by the $P$ values.

Riley and Bosland, 1998) of V. dahliae. Both types of propagules play significant roles in the life cycle of $V$. dahliae and can initiate plant infection. Whereas microsclerotia serve as survival structures, conidia have been shown to be the propagules responsible for population increases in microsclerotialinfested soil (Farley et al., 1970). Soil infestation with conidia was used in this study and the levels selected were based on previous studies (Riggs and Graham, 1995). The two levels of conidial inoculum used were comparable in terms of their effects on the

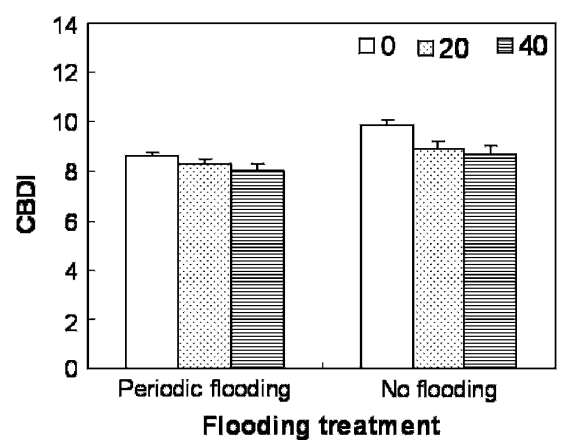

Fig. 3. Effect of periodic flooding and no flooding and inoculation of chile pepper 'AZ20' with Verticillium dahliae at three inoculum levels on chlorophyll-based difference index (CBDI). Columns are the means for each treatment (combined data from two experiments) and bars on the columns are the SES of the means. There were three inoculum levels of $0,2 \times$ $10^{7}(20$ million $)$, and $4 \times 10^{7}$ (40 million) conidia per pot, which were designated 0,20 , and 40 , respectively. There was no significant interaction between flooding treatment and inoculation $(P=0.4075)$. Probability of least squares means difference ( $P$-diff) between periodic flooding and no flooding was 0.004 . The $P$-diff values among inoculum levels $(0$, 20, and 40) were as follows: $P$-diff $(0$ and 20$)=$ $0.015 ; P$-diff $(0$ and 40$)=0.0013$; and $P$-diff $(20$ and 40$)=0.3658$.

20- and 40-d flooding treatments. Inhibition of microsclerotia production was attributed to decreased concentration of $\mathrm{O}_{2}$ and increased concentration of $\mathrm{CO}_{2}$ in flooded soils (Ioannou et al., 1977a).

The two experiments described in this study were in congruence with regard to the effects of periodic flooding and no flooding on Verticillium wilt development. In general, periodic flooding and inoculation with $V$. dahliae were accompanied by decreased plant dry weight and decreased levels in spectral reflectance-based physiological indices such as PRI and CBDI. However, it is not known why MSI and WBI were not significantly affected by flooding and inoculation treatments. Plant stress from periodic flooding was equivalent to plant stress from inoculation based on plant growth and spectral reflectance-based physiological indices. Despite the plant stress caused by periodic flooding, disease severity was greater under no flooding than under periodic flooding.

The mechanisms underlying the increase of Verticillium wilt under no-flooding conditions compared with periodic flooding are not known. Although changes in population density of $V$. dahliae were not assessed in this study, it is plausible that periodic flooding affected inoculum density of $V$. dahliae as reported under continuous flooding (Ioannou et al., 1977a; Pullman and DeVay, 1982). It is also conceivable that the activity of $V$. dahliae was impeded under periodic flooding as reflected in the fact that the decrease in plant dry weight in noninoculated plants was within the range recorded for plants inoculated with $V$. dahliae. Under no-flooding conditions, it is possible that short-term soil 
moisture fluctuation enhanced population levels and activity of $V$. dahliae. Farley et al. (1970) have shown that conidia production by $V$. dahliae increased in soil infested with microsclerotia and subjected to repeated cycles of air-drying and remoistening with soil moisture fluctuation between $4 \%$ and $50 \%$ field capacity.

An important factor that was not addressed in this study is the contribution of soil microflora to the differential response of chile pepper to $V$. dahliae under periodic flooding and no-flooding conditions. Although such contribution may be negligible in this greenhouse study because sterilized soil was used in all experiments, it is plausible that under field conditions, soil microflora may affect population levels and activity of $V$. dahliae. Ioannou et al. (1977b) have reported that in saturated soil activity of antagonistic soil, microflora may increase, whereas in nonsaturated soil, antagonistic activity of soil microflora may be decreased.

In addition to validating field observations and field surveys on increased Verticillium wilt of chile pepper under drip irrigation compared with furrow irrigation, this study showed that plant growth and physiology were more reduced under periodic flooding than under no-flooding conditions. However, it is not known to what extent these results reflect changes in plant growth and physiology under field conditions. There are no previous field data available for comparison. This area merits further research.

The information emanating from this study should be useful to chile pepper producers in understanding the ecology of $V$. dahliae with respect to furrow irrigation and drip irrigation as represented here by periodic flooding and no flooding, respectively. It appears that furrow irrigation may alleviate the impact of Verticillium wilt in fields infested with $V$. dahliae. Recommendations to implement furrow irrigation for control of Verticillium wilt are to be gauged on whether a given production field is solely infested with $V$. dahliae. These recommendations may not be tenable in production fields with a history of Phytophthora blight. In such production fields, implementing furrow irrigation would be laden with the risk of increasing Phytophthora blight. Producers will have to determine whether Phytophthora blight or Verticillium wilt is the most significant problem they are facing and apply information from this study and others to resolve the problem to their desired satisfaction.

\section{Literature Cited}

Bejarano-Alcázar, J., M.A. Blanco-López, J.M. Melero-Vara, and R.M. Jiménez-Díaz. 1996. Etiology, importance, and distribution of Verticillium of cotton in southern Spain. Plant Dis. 80:1233-1238.

Bhat, R.G. and K.V. Subbarao. 1999. Host range specificity in Verticillium dahliae. Phytopathology 89:1218-1225.

Biles, C., D.L. Lindsey, and C.M. Liddell. 1992. Control of Phytophthora root rot of chile peppers by irrigation practices and fungicides. Crop Protection 11:225-228.

Brunner, E., S. Domhof, and F. Langer. 2002. Nonparametric analysis of longitudinal data in factorial experiments. John Wiley \& Sons, Inc., New York.

DeVay, J.E., B.L. Weir, R.J. Wakeman, and J.J. Stapleton. 1997. Effects of Verticillium dahliae infection of cotton plants (Gossypium hirsutum) on potassium levels in leaf petioles. Plant Dis. 81:1089-1092.

Farley, J.D., S. Wilhem, and W.C. Snyder. 1970. Repeated germination and sporulation of microsclerotia of Verticillium albo-atrum in soil. Phytopathology 61:260-264.

Gamon, J.A. and H. Qui. 1999. Ecological applications of remote sensing at multiple scales, $\mathrm{p}$. 805-846. In: Pugnaire, F.I. and Valladares, F. (eds.). Handbook of functional plant ecology. Marcel Dekker, New York.

Gomez, K.A. and A.A. Gomez. 1984. Statistical procedures for agricultural research. 2nd Edition. John Wiley \& Sons, Inc, New York.

Hunt, E.R. and B.N. Rock. 1989. Detection of changes in leaf water content using near-infrared and middle-infrared reflectance. Remote Sensing Environ. 30:43-54.

Ioannou, N., R.W. Schneider, and R.G. Grogan. 1977a. Effect of flooding on the soil gas composition and the production of microsclerotia by Verticillium dahliae in the field. Phytopathology 67:651-656.

Ioannou, N., R.W. Schneider, R.G. Grogan, and J.M. Duniway. 1977b. Effect of water potential and temperature on growth, sporulation, and production of microsclerotia by Verticillium dahliae. Phytopathology 67:637-644.

Kuan, T.L. and D.C. Erwin. 1980. Predisposition effect of water saturation of soil on Phytophthora root rot of alfalfa. Phytopathology 70:981-986.

Pegg, G.F. and B.L. Brady. 2002. Verticillium wilts. CABI Publishing, CAB International, Wallingford, UK.

Penuelas, J., J. Llusia, J. Pinol, and I. Filella. 1997. Photochemical reflectance index and leaf pho- tosynthetic radiation-use-efficiency assessment in Mediterranean trees. Int. J. Remote Sens. 18:2863-2868.

Pullman, G.S. and J.E. DeVay. 1982. Effect of soil flooding and paddy rice culture on the survival of Verticillium dahliae and incidence of Verticillium wilt in cotton. Phytopathology 72:1285-1289.

Riggs, J.L. and C.J. Graham. 1995. A screening of New Mexico Verticillium dahliae isolates for cross-infectivity to cotton and chile, $\mathrm{p}$. 218-221. In: 1995 Beltwide Cotton Conference, 4-7 Jan, San Antonio, TX.

Riley, M.K. and P.W. Bosland. 1998. Commercial planting media effective in screening for Verticillium wilt of Capsicum anпuит. HortScience 33:285-286.

Ristaino, J.B. and S.A. Johnston. 1999. Ecologically based approaches to management of Phytophthora blight on bell pepper. Plant Dis. 83:1080-1089.

Sanogo, S. and J. Carpenter. 2006. Incidence of Phytophthora blight and Verticillium wilt within chile pepper fields in New Mexico. Plant Dis. 90:291-296.

Sanogo, S. and M. Clary. 2003. Pathogenicity on chile pepper of Verticillium dahliae recovered from three weed hosts in New Mexico. Plant Dis. 87:450.

Skaggs, R., M. Decker, and D. VanLeeuwen. 2000. A survey of southern New Mexico chile producers: Production practices and problems. New Mexico Agricultural Experiment Station Technical Bulletin, No. 782.

Tomescu, A., V. Voican, and V. Racaru. 2000. Some physiological changes induced by the fungus Verticillium dahliae in pepper plants, p. 151-154. In: Tjamos, E.C., R.C. Rowe, J.B. Heale, and D.R. Fravel (eds.). Advances in Verticillium research and disease management. APS Press, St. Paul, MN.

Tsor (Lakim), L., O. Erlich, S. Amitai, and M. Hazanovsky. 1998. Verticillium wilt of paprika caused by a highly virulent isolate of Verticillium dahliae. Plant Dis. 82:437-439.

USDA-NASS. 2007. Quick stats (vegetables) online. U.S. Department of Agriculture-National Agricultural Statistics Service.

Wilhem, S. 1955. Longevity of the Verticillium wilt fungus in the laboratory and field. Phytopathology 45:180-181.

Woolliams, G.E. 1966. Host range and symptomatology of Verticillium dahliae in economic, weed, and native plants in interior British Columbia. Can. J. Plant Sci. 46:661669.

Xie, J., E.S. Cardenas, T.W. Sammis, M.M. Wall, D.L. Lindsey, and L.W. Murray. 1999. Effects of irrigation method on chile pepper yield and Phytophthora root rot incidence. Agric. Water Manage. 42:127-142. 